Article

\title{
Biodegradable Foam Cushions as Ecofriendly Packaging Materials
}

\author{
Joo Seong Sohn $₫$, Hyun Keun Kim, Shin Won Kim, Youngjae Ryu® and Sung Woon Cha * \\ School of Mechanical Engineering, Yonsei University, Seoul 03722, Korea; ssamjjang87@yonsei.ac.kr (J.S.S.); \\ sagegny@yonsei.ac.kr (H.K.K.); 0shinmy0@yonsei.ac.kr (S.W.K.); yjryu1027@yonsei.ac.kr (Y.R.) \\ * Correspondence: swcha@yonsei.ac.kr; Tel.: +82-2-2123-7224
}

Received: 7 February 2019; Accepted: 19 March 2019; Published: 21 March 2019

\begin{abstract}
In this work, a high-magnification extrusion-foaming technique for biomass-based biodegradable composite materials using water vaporization was examined. Starch was selected as the biomass and polylactic acid was selected as a biodegradable matrix resin. No additional plasticizer or additives were used in this extrusion-foaming process. The foaming ratio was deduced according to the conditions of the extrusion-foaming process to confirm the forming characteristics of the foaming materials. Scanning electron microscopy was performed to examine the morphology of the composite foam. To investigate the potential of the foam cushion as an ecofriendly packing material, we conducted experiments on its static compression and dynamic cushioning properties and examined whether its biodegradability could be controlled by varying the mixing ratio of the materials. Thus, we developed a water-foaming process that is ecofriendly and whose products can be recycled as compost after use.
\end{abstract}

Keywords: biomass; starch; polylactic acid; extrusion; foaming process; foam cushion

\section{Introduction}

As environmental regulations such as disposal charges for nondegradable packaging materials and environmental improvement charges are becoming stricter, consumer demand for ecofriendly products is increasing. Therefore, research and development related to biomass-based materials are actively performed throughout the world. Because biomass is a carbon dioxide $\left(\mathrm{CO}_{2}\right)$ sink, it can contribute to the reduction of greenhouse gases when recycled and can be used as a substitute material for many petroleum plastics, having the advantages of being biodegradable as well as harmless to the human body. As a natural resource, biomass is abundant and inexpensive, offering cost-competitiveness. Recently, composite materials based on biomass have been widely used as ecofriendly lightweight alternatives in electronic and packaging materials. The objective of this study was to develop biodegradable cushioning materials for packaging to safeguard products from external shocks by studying natural plant-derived composite materials. The main process for the development of cushioning materials is the extrusion-foaming process, which produces cells in the material during the plastic-manufacturing process and uniformly disperses them into the product. The most important aspect of the foaming process is controlling the size, shape, and distribution of the cells. The foaming process has the disadvantage of being more variable and difficult to control than other processes but can achieve product features, such as lightness and heat insulation, that cannot be obtained via other widely used processes. In general, foaming technology can be divided into chemical foaming and physical foaming. In chemical foaming, the chemical blowing agent is chemically decomposed by heat, which is harmful to the environment. Physical foaming is disadvantaged in that expensive additional equipment is needed to inject the foaming agent, such as $\mathrm{CO}_{2}$ or $\mathrm{N}_{2}$, into the cylinder. Studies on the implementation of foam using biomass as a substitute for petroleum-based plastic foam 
have mainly used $\mathrm{CO}_{2}, \mathrm{~N}_{2}$, and-in rare cases-supercritical fluid gas [1-6] or a microwave heating process $[7,8]$. Thus, the objective of the present study was to develop an ecofriendly high-magnification foaming technology that does not require additional equipment. It involves an extrusion water-foaming process using vaporization phenomena. With regard to materials, it is essential to develop a new biodegradable cushioning packaging material to replace the existing nondegradable polystyrene and polyethylene packaging materials, which contribute to environmental pollution. Thus, in this study, we developed biodegradable high-magnification foam composites with twofold (or greater) volume expansion using starch and polylactic acid (PLA). Starch was employed as the main ingredient in the foaming process using plant-derived natural materials. This is because, similarly to thermoplastic polymer resin, when starch is heated above a certain temperature gelatinization occurs and material flow is possible. Starch is composed of amylose, which is a linear polymer, and amylopectin, which is a branched polymer. When starch is added to water and heated, the regular micelle structure of the starch is loosened by heat energy and the water penetrates the structure, which causes swelling. When the heating is continued, the micelle structure is destroyed and a colloidal solution is formed, increasing the viscosity [9]. Thus, we induced high-magnification foaming by exploiting the material properties of starch and the vaporization of water.

\section{Materials and Methods}

\subsection{Materials}

In this study, waxy corn starch (Samyang Genex Co., Ltd., Seoul, Korea) with a high amylose content $(60 \%)$ was used to achieve a sufficient foaming ratio for satisfying the basic properties of biodegradable cushioning materials. The starch was highly purified by separating and removing the embryo, corn bran, and gluten, etc., from the waxy corn. PLA (Nature Works Inc., Minnesota, USA, grade no. Ingoe 8052D), a biodegradable and thermoplastic aliphatic polyester derived from renewable resources, such as corn starch, potatoes, or sugarcane, was also used.

\subsection{Method}

\subsubsection{Extrusion-foaming Process Conditions}

This study was performed using an extrusion machine which had a $40 \phi$ miniature extruder and a motor with an output of $7.5 \mathrm{~kW}$ and a maximum speed of 1740 revolutions per minute (RPM). The driving unit of the extruder included a motor as a power source for rotating the screw and a power-transmitting unit. Because the reduction ratio was 15:1, the screw could be adjusted from 0 to 116 RPM. The pressure and discharge amount were controlled through modification of the motor speed. The heater was divided into four parts with respect to the longitudinal direction of the barrel and the temperature of the nozzle was controlled in five parts. Thus, the flow of molten material was controlled by manipulating the temperature corresponding to the five parts of the extruder. The temperatures of the first and second parts of the barrel and the nozzle were increased to 90, 120, 150 , and $180{ }^{\circ} \mathrm{C}$. The temperatures of the third and fourth parts of the barrel were fixed at $70{ }^{\circ} \mathrm{C}$, which is the starch gelatinization temperature. Because natural materials are vulnerable to high temperatures, carbonization was considered. In the die part, a pressure drop occurred in the starch that was gelatinized through the barrel. During the process of the material passing through the die, a pressure drop from a high pressure to a low pressure occurred, and water (as a blowing agent) was vaporized. This phase change was used to induce high-magnification foaming. The flowability and pressure distribution of the material were determined by the shape of the screw inside the barrel. The shape of the screw was determined by the pitch of the screw, the arrangement of the blades, and the gap of the blades, etc. 


\subsubsection{Induction of High-magnification Foaming}

Figure 1 presents the extruder and auxiliary equipment used for the foaming in this study. The moisture added to the starch was vaporized owing to the processing temperature of the die. When the material was discharged through the die, a phase change occurred owing to the rapid pressure drop, forming cells in the material. The process of this experiment can be divided into three stages. In the first stage, a certain amount of water was added to the starch to increase the water content for high-expansion foaming. In the second stage, starch and PLA were mixed at a specific ratio. In the third stage, the composite material was foamed via an extrusion process. The content of water (as a blowing agent) was fixed at $20 \%$ according to the results of a preliminary experiment (Figure 2). When the water content was $40 \%$, a dough-type mixture was formed when the starch and PLA were mixed. This caused problems in supplying the material to the hopper throughout the whole process, resulting in the deterioration of the workability and an unstable shape due to excessive moisture generated in the resultant product. When the water content was $30 \%$, a small lump was formed upon mixing, and the material could be supplied to the hopper more easily than in the case of a water content of $40 \%$. However, the product obtained had an excessive moisture content and was not suitable for the foaming process. When the water content was $20 \%$, the material was easily supplied to the hopper because it was in powder form, similar to that before the addition of water, and the discharged extrudate had an appropriate moisture content for the other experimental conditions. The results of this preliminary experiment indicated that if the water content is low, the viscosity is high, and foaming does not occur. On the contrary, if the water content is too high, excessive steam may be generated, and cell size and distribution of the foam may be uneven. Additionally, because water vapor penetrates the surface of the foam, the foaming ratio may be reduced, and if the water content remains excessive after the foaming process, a separate drying step may be required. The extrusion machine used in this study was equipped with a screw specially designed to achieve high-magnification foaming. The conditions of the extrusion-foaming process are presented in Table 1, including the nozzle (NZ) and heater (H1-H4) temperatures and the water content. Here, "phr" represents "parts per hundred resin." In this study, water was added on the basis of 100 parts (by weight) of the mixture of starch and PLA. The change in the foaming ratio according to the mixing ratio of starch and PLA was examined. To optimize the material and the process for obtaining a high-magnification foam, the optimal mixing ratio and water content of the main and sub-materials were studied. Moreover, for the design and fabrication of the die and screw, the process conditions, pressure drop, and material flow characteristics were considered.
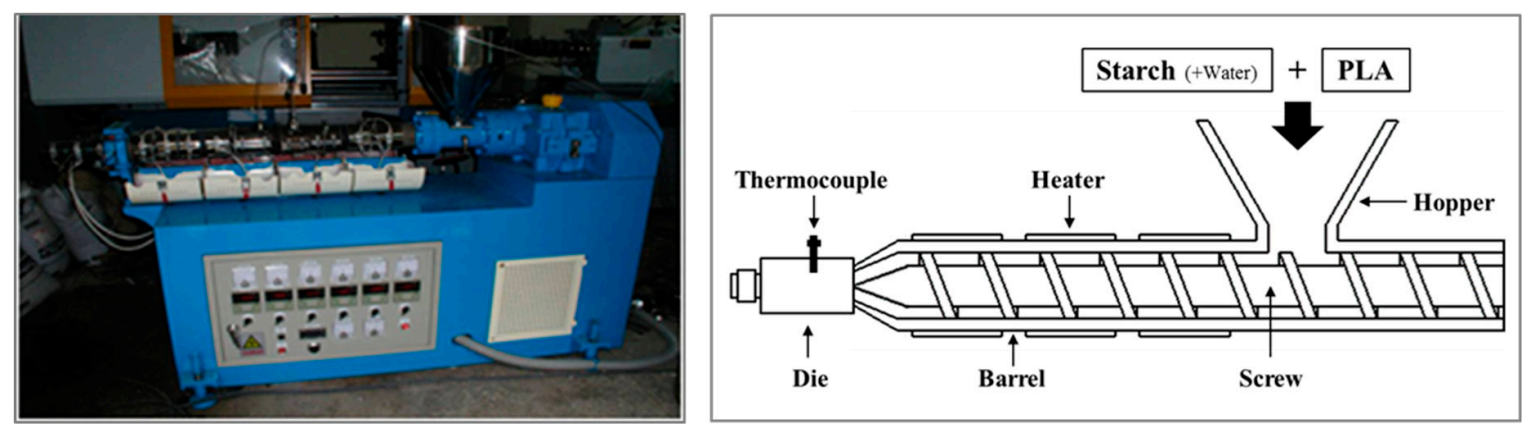

Figure 1. Extruder and auxiliary equipment used in this study. Legend: PLA, polylactic acid. 


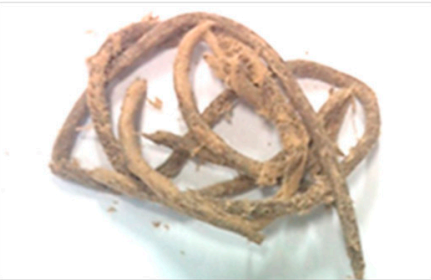

(A) Water contents $(\mathrm{phr})=\mathbf{2 0}$

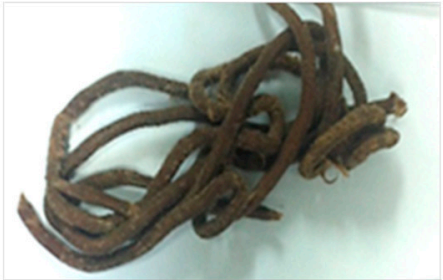

(B) Water contents $(\mathrm{phr})=\mathbf{3 0}$

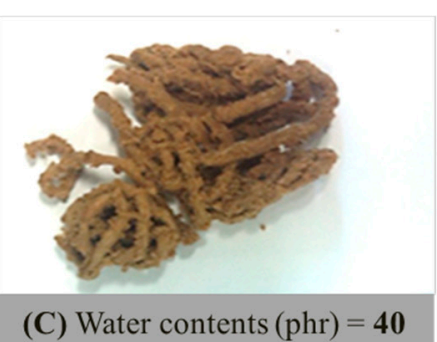

(C) Water contents $(\mathrm{phr})=\mathbf{4 0}$

Figure 2. Appearance of the virgin starch foam cushion with different water contents. Legend: phr, parts per hundred resin.

\subsubsection{Screw Design for Extrusion Foaming of Biomass-based Composite}

It was necessary to design a screw able to generate a pressure profile capable of uniform mixing and metering between materials. Additionally, it was necessary to create natural materials with low viscosity and to increase the pressure-drop rate. Thus, we designed a special screw for the extrusion of the natural material to match the material characteristics (Figure 3). The viscosity of a natural material can be controlled by changing the amount of water, but because this is generally low, a low-viscosity resin is assumed in the design. There is little need for a mixing zone, because the material is pre-mixed before being placed in a barrel. In the case of general polymer-forming screws, compression ratios of 2:1 to $4: 1$ are used. However, in this study, the compression ratio was designed to be $4: 1$ to $5: 1$ in order to induce high-magnification foaming via the pressure drop. The detailed design plan of the screw used in this study is as follows. The feeding zone, which is a solid-conveying zone, occupies $10 \%-20 \%$ of the total length and has dimensions of $140-280 \mathrm{~mm}$. The pre-mixing zone for the natural material was designed to be short because it does not need a long feeding zone. The compressing zone occupies $>50 \%$ of the total length and was designed twice over the compressing zone by distributing the discrete compressing zone considering the motor torque and natural carbonization. The mixing zone is supplied with pre-mixed natural material; thus, it does not need a mixing feature but is designed to employ Egan-type mixing, which is a simple feature considering the dispersion and distribution of water and natural materials. Finally, the channel depth was designed to be small and constant so that the plasticized resin can pass through the die.
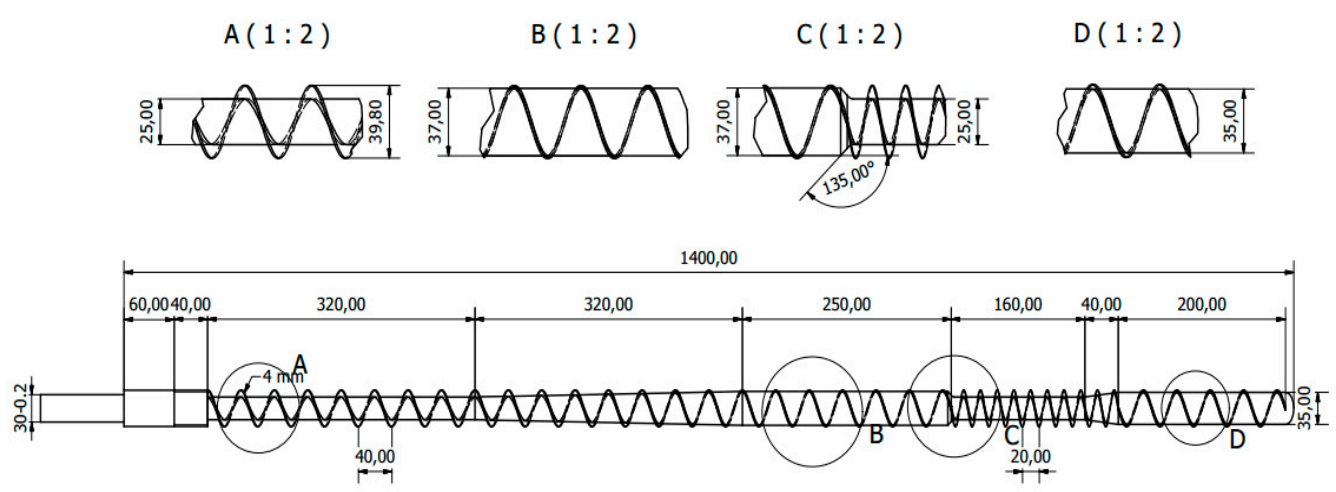

Figure 3. Detailed drawings of the screw for the extrusion foaming of biomass-based composites.

\subsubsection{Die Design for Extrusion Foaming of Biomass-based Composites}

The process conditions, such as the die temperature, barrel temperature, foaming agent, and gas input amount, affect the foaming ratio of the foam when the foaming process is performed using the extruder, but the die shape of the extruder has the greatest influence on the foaming ratio. The design of the die was developed according to the basic properties of the material, such as the viscosity. In this study, the die needed to be designed to maximize the pressure-drop rate considering the viscosity of the composite materials (starch and PLA) in order to obtain 
high-magnification foam. Generally, in designing a die, it is necessary to uniformly form a molten resin kneaded in a barrel, and if possible, the die should not be affected by the fluidity of the resin. Additionally, temperature control must be possible. Figure 4 shows photographs and a design drawing of the die used in this study. The die consisted of three parts, as shown in Figure 5.

In Figure 5, (a) shows the basic die connected to the barrel, (b) shows the adapter that connects the basic die and the designed die, and (c) shows the core die designed in this study, which corresponds to the discharging part. The diameter of the designed die was $1 \mathrm{~mm}$, and the length was $3 \mathrm{~mm}$, which maximized the pressure drop. It was thought that the viscosity of the biomass-based composite material would decrease.
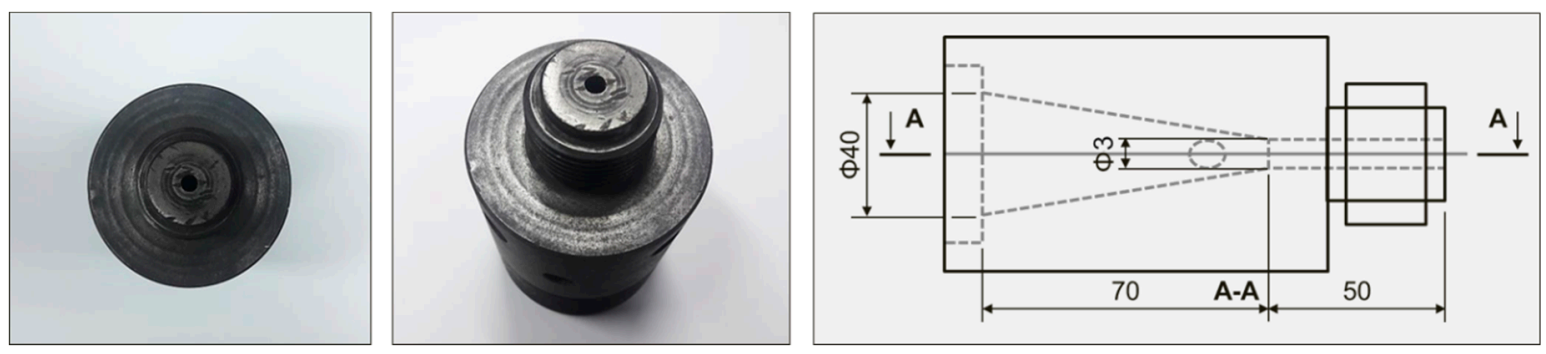

Figure 4. Photographs and design drawings of the die used in this study.

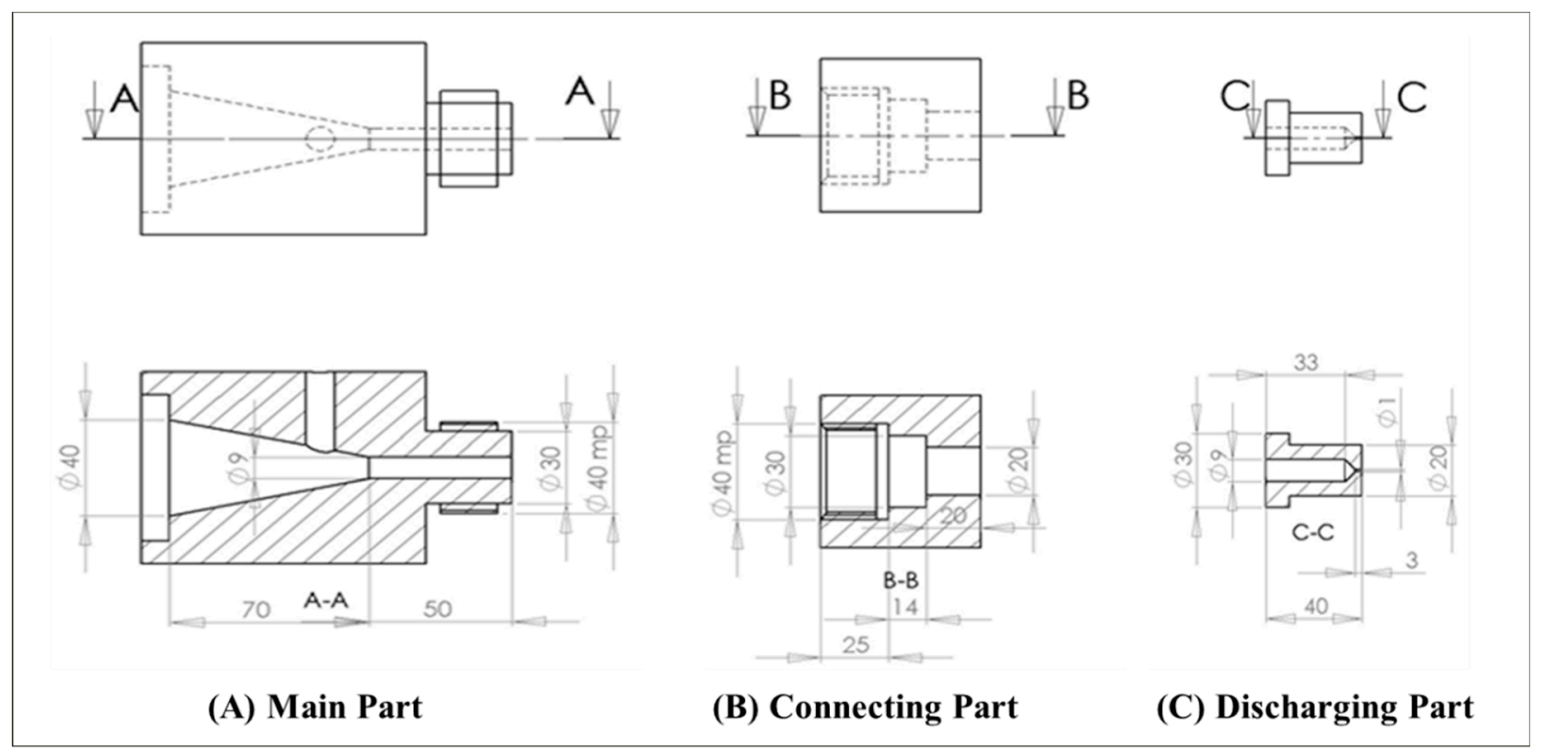

Figure 5. Detailed design drawings of the die parts for high-magnification foaming.

\section{Results and Discussion}

\subsection{Foaming Characteristics According to Temperature of Extrusion-foaming Process}

In this study, the foaming characteristics of the extrudate were determined according to the temperature of the nozzle and the barrel for $100 \%$ starch, to derive a suitable extrusion-foaming process temperature. The temperature of the barrel was set as $70{ }^{\circ} \mathrm{C}$, which is the gelatinization temperature of starch, and the temperatures of the barrel and nozzles of the upper parts ( 1 and 2$)$ were increased to $90-150{ }^{\circ} \mathrm{C}$ (Table 1 and Figure 6). At a temperature of $\leq 90{ }^{\circ} \mathrm{C}$, the viscosity of the material was too high; thus, the molten composition was not discharged normally from the die. The discharge was normal at $150{ }^{\circ} \mathrm{C}$. As the temperature increased from $90^{\circ} \mathrm{C}$ to 120 and $150{ }^{\circ} \mathrm{C}$, the foaming rate increased. At $180^{\circ} \mathrm{C}$, the foaming rate decreased after the foaming and cooling of the starch. Thus, the extrusion-foaming characteristics confirmed that the most suitable process temperature was $150{ }^{\circ} \mathrm{C}$ (Figure 7). 


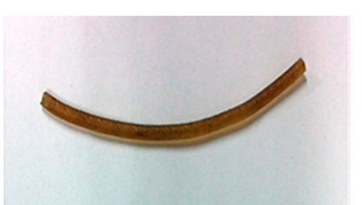

Cond. 1

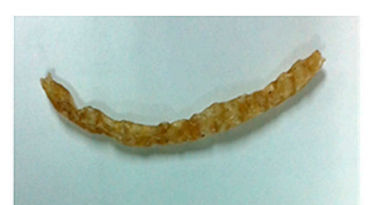

Cond. 2

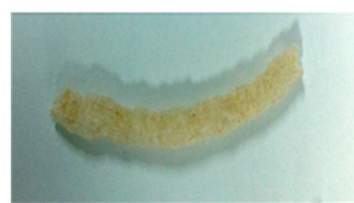

Cond. 3

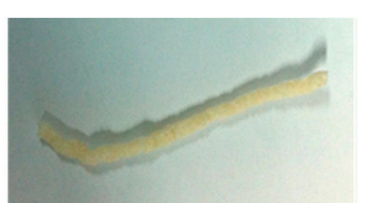

Cond. 4

Figure 6. Appearance according to the water content of the virgin starch foam cushion: Cond. $1,90^{\circ} \mathrm{C}$; Cond. $2,120^{\circ} \mathrm{C}$; Cond. $3,150{ }^{\circ} \mathrm{C}$; Cond. $4,180^{\circ} \mathrm{C}$.
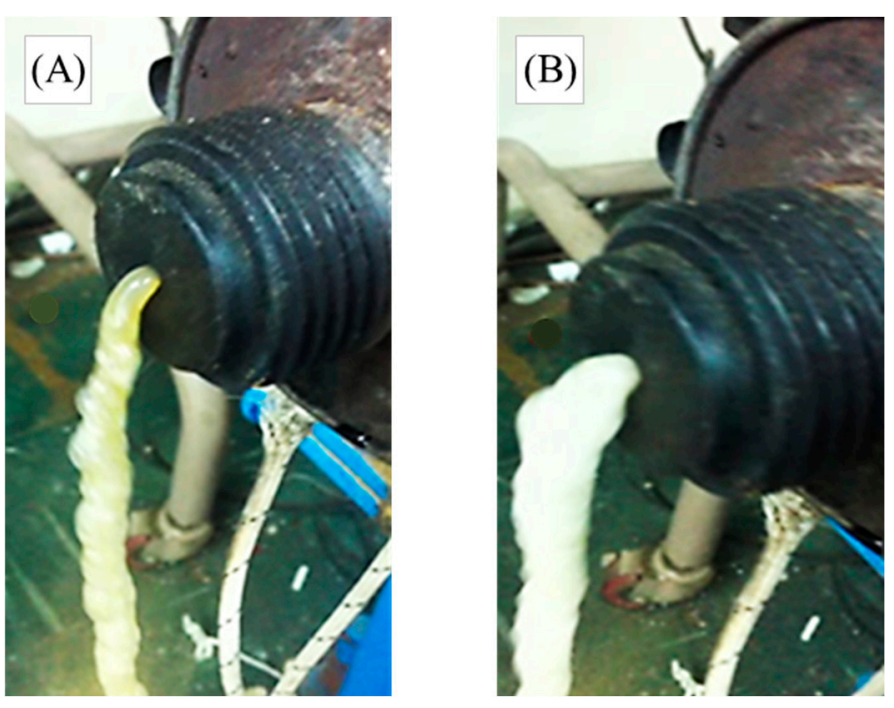

Figure 7. Discharge characteristics of the composite melt according to the extrusion process temperature: (a) $120^{\circ} \mathrm{C}$; (b) $150^{\circ} \mathrm{C}$.

Table 1. Experimental conditions for the extrusion process. Legend: RPM, revolutions per minute; NZ, nozzle; $\mathrm{H}$, heater.

\begin{tabular}{|c|c|c|c|c|c|c|}
\hline Material & \multicolumn{2}{|c|}{ Starch } & \multicolumn{3}{|c|}{ PLA } & \\
\hline Die Diameter $(\phi)$ & \multicolumn{6}{|c|}{3} \\
\hline Screw Speed (RPM) & \multicolumn{6}{|c|}{66.6} \\
\hline Water Content (phr) & \multicolumn{6}{|c|}{20} \\
\hline \multirow{5}{*}{ Temperature $\left({ }^{\circ} \mathrm{C}\right)$} & & NZ & H1 & $\mathrm{H} 2$ & $\mathrm{H} 3$ & $\mathrm{H} 4$ \\
\hline & 1 & 90 & 90 & 90 & 70 & 70 \\
\hline & 2 & 120 & 120 & 120 & 70 & 70 \\
\hline & 3 & 150 & 150 & 150 & 70 & 70 \\
\hline & 4 & 180 & 180 & 180 & 70 & 70 \\
\hline
\end{tabular}

\subsection{Foaming Ratio of Foamed Composite Cushions According to Mixing ratio of Starch and PLA}

Foams were able to be prepared when the extrusion-foaming process was performed for $100 \%$ starch, but the specimens were not suitable to be used as packaging cushioning materials because of their high hardness and brittleness. To compensate for these poor physical properties and to ensure biodegradability, a composite material was formed by adding PLA, and additional foaming experiments were conducted (Figures 8 and 9). Unfoamed specimens formed at a nozzle temperature of $90^{\circ} \mathrm{C}$, which is below the vaporization point of water, were used as a control group. The foaming ratio was derived from density measurements for these different conditions. The foaming ratio for a starch content of $100 \%$ was the highest $(97.0 \%)$, and the foaming ratio decreased as the PLA content increased. The conditions of Starch90/PLA10 and Starch80/PLA20 were determined to be suitable for cushioning 
materials, as the Starch100 specimen was fragile and vulnerable to moisture. The Starch70/PLA30 condition yielded a foaming ratio of $\leq 90 \%$, and the specimen was unsuitable as a cushioning material compared with the other experimental conditions.

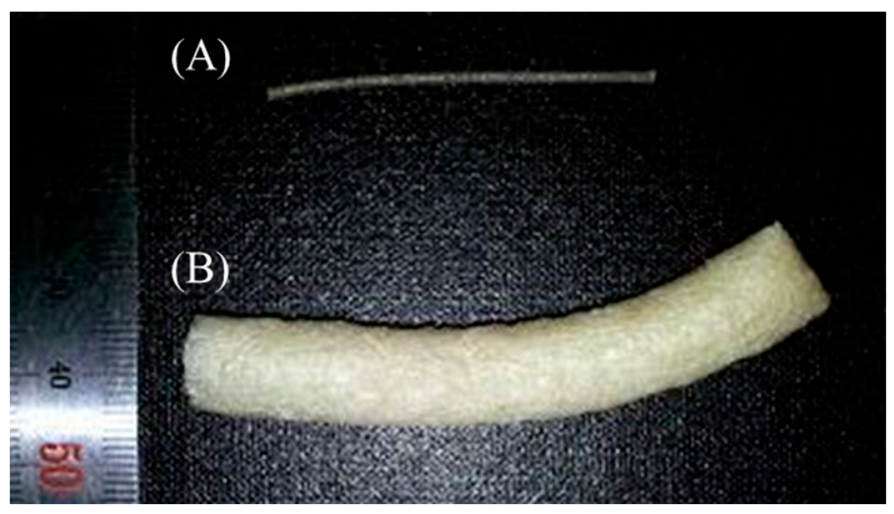

Figure 8. Unfoamed and foamed extruded materials: (a) unfoamed solid specimen; (a) foamed specimen.

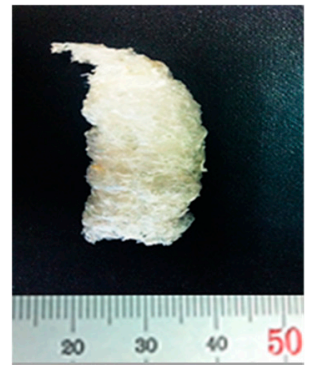

(A) Starch100/PLA0

Foaming Ratio $=97.0 \%$

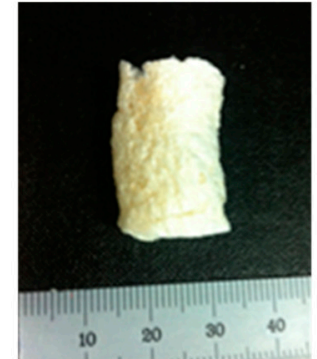

(B) Starch90/PLA10 Foaming Ratio $=93.5 \%$

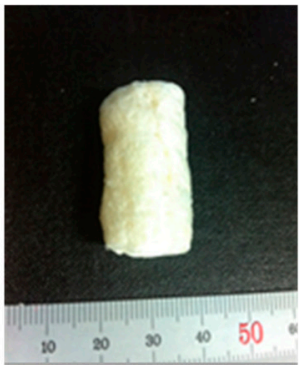

(C) Starch80/PLA20

Foaming Ratio $=94.2 \%$

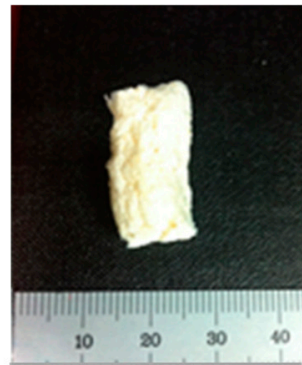

(D) Starch70/PLA30

Foaming Ratio $=89.4 \%$

Figure 9. Appearance and foaming ratio of composite foam cushions with respect to the mixing ratio of starch and PLA.

\subsection{Static Compression Characteristic}

The static compression characteristics were measured according to the KS A 1030 standard for examining the resistance of the biomass-based packing buffer to external stress. The developed cushioning material was compressed using a universal testing machine (QMESYS Co., Ltd., Gunpo, Korea, Model No. QM-100T) measuring device with a plate-type compression mold used to measure the stress and load per unit displacement. The compression rate was fixed at $10 \mathrm{~mm} / \mathrm{min}$ and the load was measured according to the displacement. When the static compression characteristics were measured according to the mixing ratio of starch and PLA, the tendency shown in Figure 10 was observed. As the PLA content increased, the static compression characteristic value increased. 


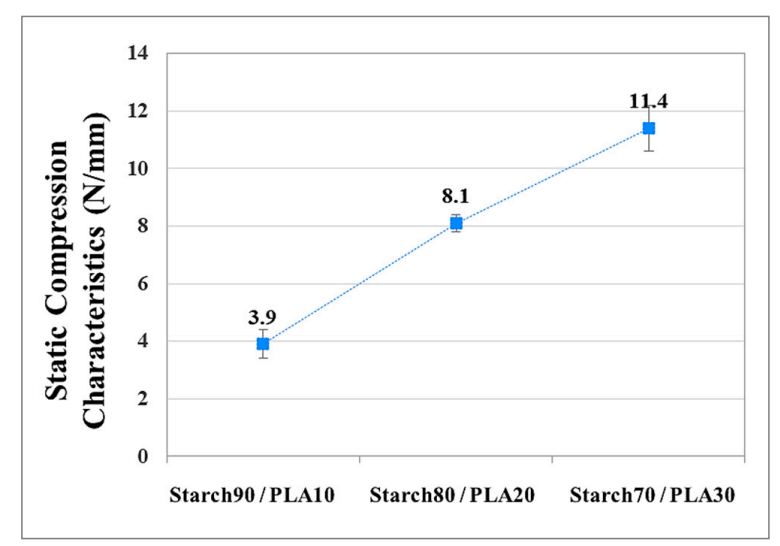

Figure 10. Measurement results for the static compression characteristics of foamed cushioning materials with different mixing ratios of starch and PLA.

\subsection{Dynamic Cushioning Characteristics}

To investigate the dynamic cushioning characteristics, the physical properties were evaluated according to ASTM D 3332. This standard is used for measuring the acceleration when a plate-shaped object of $1 \mathrm{~kg}$ is dropped from a height of $50 \mathrm{~cm}$. The prepared cushioning-material specimen was placed between the plate-type object and the bottom, and the change in the acceleration was measured. The dynamic cushioning characteristics, including the impulse and the maximum acceleration of the cushioning-material specimen, were measured. In the experiment, a three-axis accelerometer was used, and the acceleration was calculated using units of $1000 \mathrm{~Hz}$ and $1 \mathrm{~ms}$. Figure 11 shows the tendency of the acceleration according to the mixing ratio of starch and PLA. The impulse (Figure 12a) was obtained from the integrated value and the maximum acceleration (Figure 12b) at that time was measured. Figure $12 \mathrm{~b}$ shows a graph indicating the maximum acceleration according to the mixing ratio of starch and PLA. In the case of the maximum acceleration, the maximum force was applied to the cushioning material by the fall of a plate-type object of $1 \mathrm{~kg}$ (constant mass). In the experiment, the maximum acceleration was the lowest for Starch90/PLA10 and the highest for Starch80/PLA20. Because the specimen obtained under the Starch90/PLA10 condition exhibited the lowest acceleration, this cushioning material received the lowest maximum force. The impulse according to the mixing conditions of starch and PLA was derived from data calculated by multiplying the maximum velocity (obtained by integrating the acceleration) by the mass of the impacting object. In the experiment, the impulse tended to increase as the starch content decreased. Samples with a higher starch content are considered to have better cushioning properties because they have a higher foaming ratio and more cells.

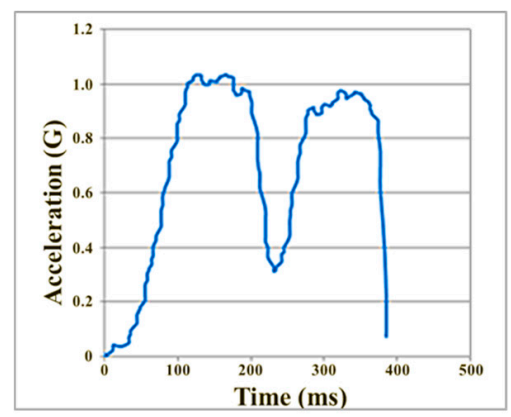

(A)

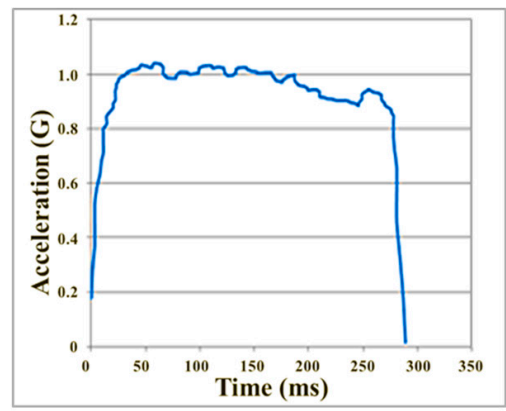

(B)

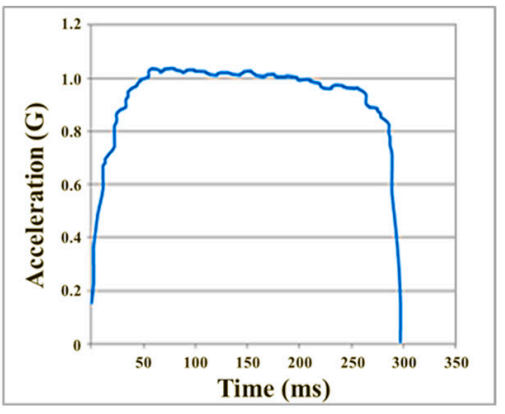

(C)

Figure 11. Measurement results for the dynamic cushioning characteristics of the foam cushion according to the mixing ratio of starch and PLA: (a) Starch90/PLA10; (b) Starch80/PLA20; (c) Starch70/PLA30. 


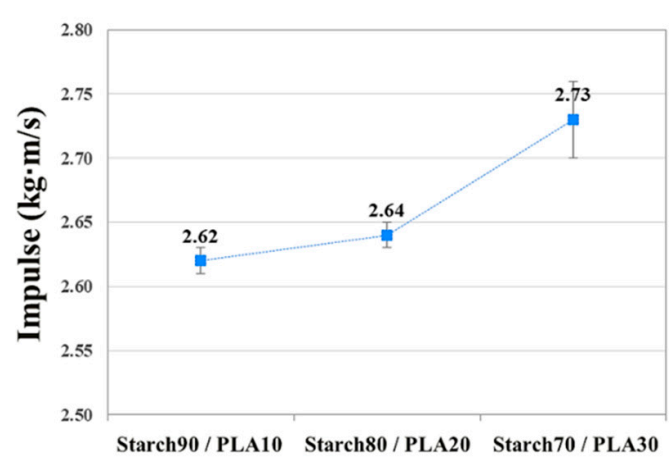

(A)

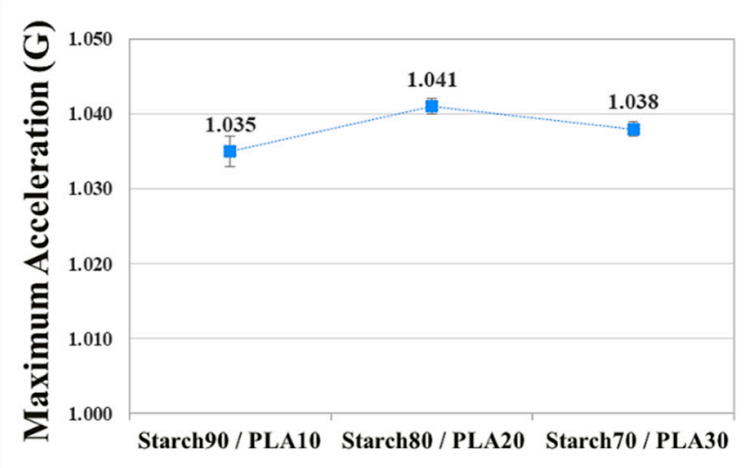

(B)

Figure 12. Measurement results for the (a) impulse and (b) maximum acceleration of foamed cushioning materials with different mixing ratios of starch and PLA.

\subsection{Scanning Electron Microscopy (SEM) Image Analysis of Extruded Foam}

SEM images were obtained to analyze the morphology of the foam, and the magnifications were $\times 60$, $\times 120, \times 1500$, and $\times 4000$. Figure 13 shows that for the $100 \%$ starch condition, the cell wall was relatively well-formed, and high-magnification foaming was induced. However, the foam was brittle and thus unsuitable for use as a cushioning material. The cell wall was thin-similar to vinyl-in the condition where the starch and PLA were mixed and foamed. The starch:PLA mixing ratio of 80:20, which is close to 90:10, resulted in a relatively compact structure. By comparison, for the mixing ratio of 70:30, the cell-wall thickness increased.

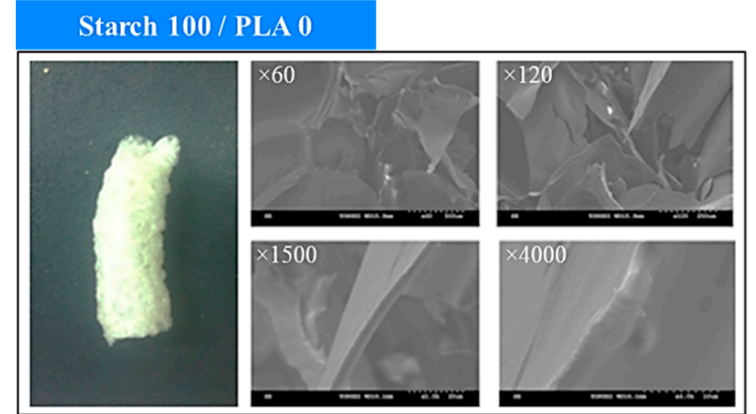

Starch 80 / PLA 20
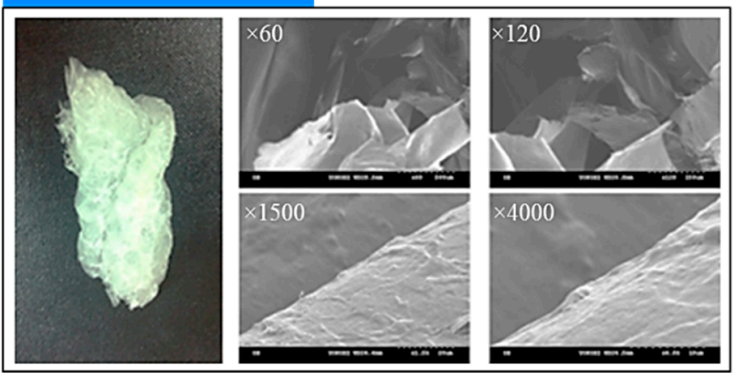

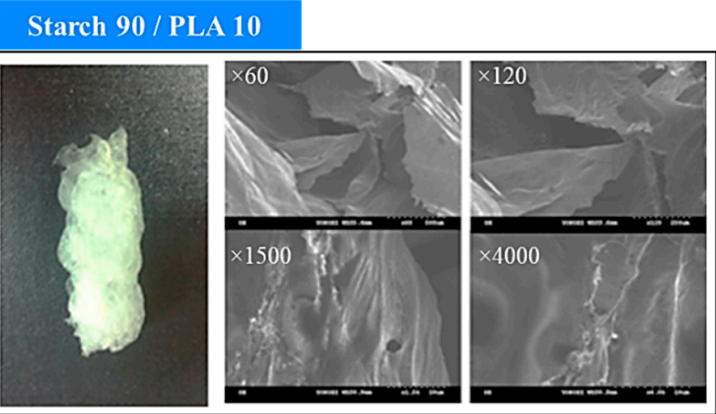

Starch 70 / PLA 30

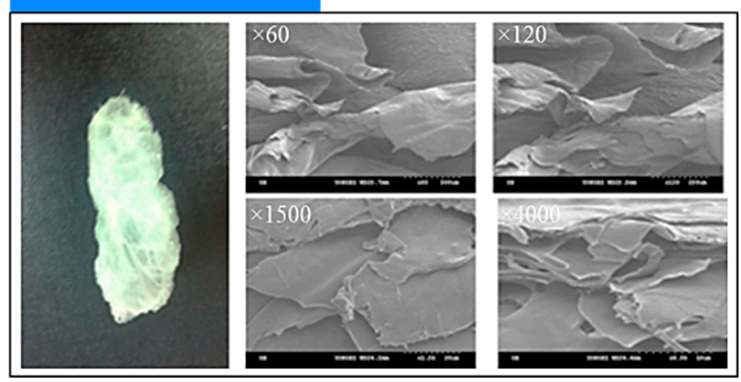

Figure 13. Cross-sectional scanning electron microscopy (SEM) images of the composite foam according to the mixing ratio of starch and PLA. 


\subsection{Biodegradability Control of Biomass-based Composite Foam Cushion}

Biodegradability measurements were performed according to KSM3100-1, a Korean industrial standard (Figure 14). The sample was buried $30 \mathrm{~cm}$ below the ground and its weight loss measured over a period of five weeks. This test was intended to elucidate the possibility of controlling the biodegradability in a landfill environment by changing the mixing ratio of starch and PLA (Figure 15). Biodegradation of biomass cushioning materials occurs at a high rate, which may cause problems in product circulation and commercialization. The biodegradability of the starch and PLA composite foam cushioning material was higher with higher starch contents. The results of the experiment confirmed that the biodegradability can be controlled by changing the mixing ratio of the materials. Thus, for commercialization, the biodegradability can be adjusted according to the circulation time from the use of the material to its disposal by the consumer. It is believed that starch, which is a natural material with a high degree of biodegradability, decomposes first, and that PLA, which is closely mixed in the composite material, decomposes partially. In a previous study, Kozlowski et al. observed that the initial cracks in starch/PLA composites were caused by starch separating from the matrix first [10]. Starch-based foam has excellent biodegradability compared with the conventional solid unit because of its large contact area with the environment, which allows for biodegradation by microorganisms.

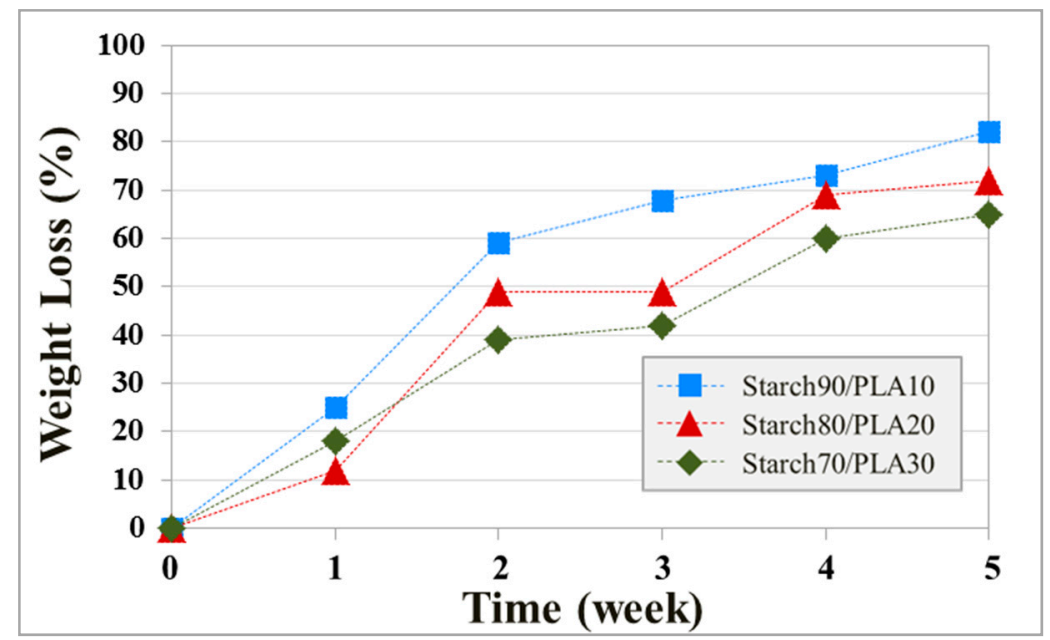

Figure 14. Biodegradation tendency of the biomass-based composite foam cushion. 

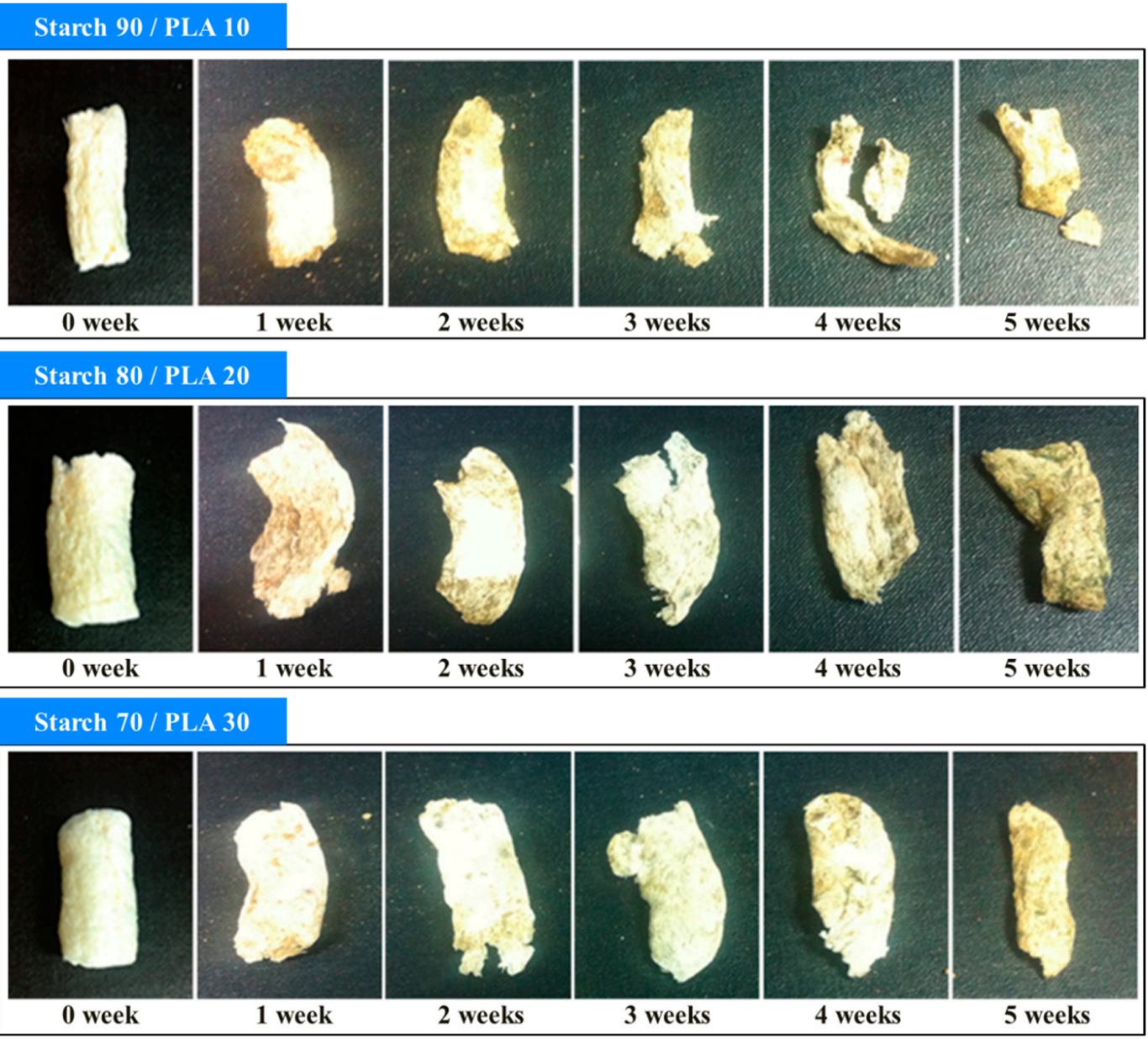

Figure 15. Results of biodegradation measurements of the biomass-based composite foam cushions over time.

\section{Conclusions}

In this work, we developed an ecofriendly high-magnification extrusion-foaming technology using the vaporization phenomenon of water for obtaining biomass-based biodegradable composite cushioning materials (Figure 16).
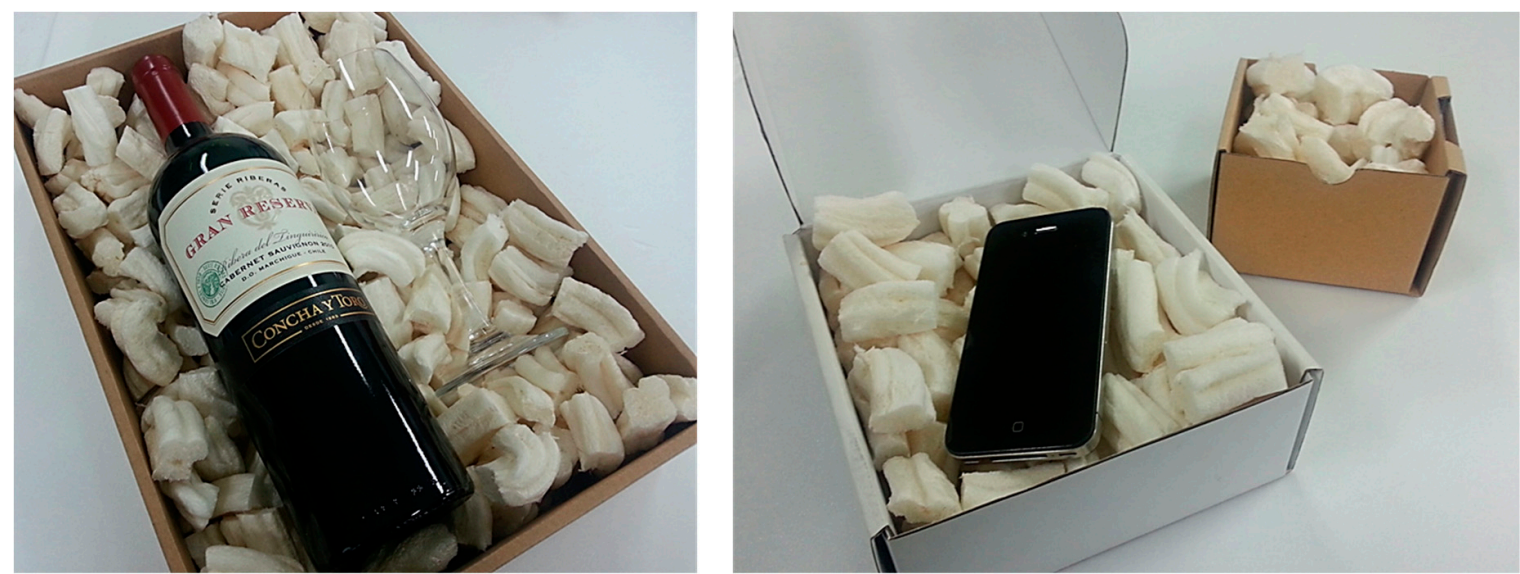

Figure 16. Use of the starch-based biomass composite cushioning material prepared in this study. 
The cushioning properties of the composite materials are due to the cells in the independent space inside the foam, with the impulse decreasing as the starch content increases. However, if the PLA content is too low, the composite is prone to crumbling; thus, an appropriate mixing ratio should be employed. In the present study, Starch90/PLA10 and Starch80/PLA20 were identified as the most suitable mixing ratios for obtaining a packing material, considering the foaming ratio and cushioning properties.

The die designed in this study expanded and caused a sudden pressure drop. Additionally, by inducing the gelatinization of the starch, the viscosity was increased, and, simultaneously, the vaporization of water led to high-magnification foaming. This process is capable of securing moldability without additional plasticizers or additives and reduces the manufacturing cost owing to its simple manufacturing method that does not require a foaming gas or chemical foaming agent. The product is more ecofriendly and less expensive than existing petrochemical synthetic resin foam products and can be decomposed by microorganisms in nature, as was confirmed by biodegradability measurements.

The proposed manufacturing process can reduce power consumption, $\mathrm{CO}_{2}$ emissions, and processing costs, as well as improve the circulation of waste resources. In addition to biomass, which was used in this study, it is possible to produce low-priced products by utilizing other plant byproducts, such as wood flour, wheat bran, rice straw, rice bran, and hemp (Figure 17). It is expected that sustainability will be ensured by the production and consumption of biomass-based products and the realization of resource circulation in conjunction with nature.

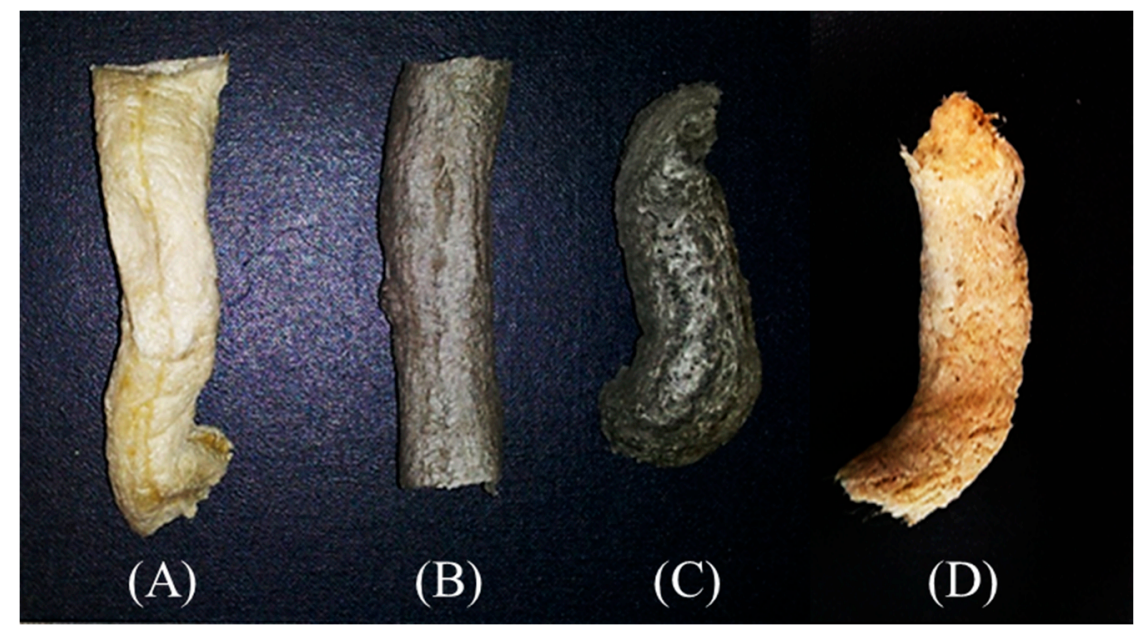

Figure 17. Biomass-based foam samples prepared via the proposed extrusion-foaming process: foam cushions of (a) wood flour and polypropylene; (b) wheat bran and polypropylene; (c) wheat bran and acrylonitrile butadiene styrene; and (d) starch, wheat bran, and hemp.

Author Contributions: Conception and design of experiments, J.S.S. and H.K.K.; execution of experiments, J.S.S. and H.K.K.; analysis of data, J.S.S and H.K.K.; data curation, S.W.K. and Y.R.; writing-original draft preparation, J.S.S.; writing—review and editing, J.S.S.; funding acquisition, S.W.C.

Funding: This research was supported by the Basic Science Research Program via the National Research Foundation of Korea (NRF), which is funded by the Ministry of Education (No. NRF-2018R1D1A1B07049405).

Conflicts of Interest: The authors declare no conflict of interest.

\section{References}

1. Alavi, S.H.; Gogoi, B.K.; Khan, M.; Bowman, B.J.; Rizvi, S.S.H. Structural properties of protein-stabilized starch-based supercritical fluid extrudates. Food Res. Int. 1999, 32, 107-118. [CrossRef]

2. Alavi, S.H.; Rizvi, S.S.H.; Harriott, P. Process dynamics of starch-based microcellular foams produced by supercritical fluid extrusion. I: Model development. Food Res. Int. 2003, 36, 309-319. [CrossRef] 
3. Cho, K.Y.; Rizvi, S.S.H. 3D microstructure of supercritical fluid extrudates I: Melt rheology and microstructure formation. Food Res. Int. 2009, 42, 595-602. [CrossRef]

4. Goel, S.K.; Beckman, E.J. Nucleation and growth in microcellular materials: Supercritical $\mathrm{CO}_{2}$ as foaming agent. AIChE J. 1995, 41, 357-367. [CrossRef]

5. Jeong, H.; Toledo, R. Twin-screw extrusion at low temperature with carbon dioxide injection to assist expansion: Extrudate characteristics. J. Food Eng. 2004, 63, 425-432. [CrossRef]

6. Muljana, H.; Picchioni, F.; Heeres, H.J.; Janssen, L.P.B.M. Supercritical carbon dioxide $\left(\mathrm{scCO}_{2}\right)$ induced gelatinization of potato starch. Carbohydr. Polym. 2009, 78, 511-519. [CrossRef]

7. Lopez-Gil, A.; Silva-Bellucci, F.; Velasco, D.; Ardanuy, M.; Rodriguez-Perez, M.A. Cellular structure and mechanical properties of starch-based foamed blocks reinforced with natural fibers and produced by microwave heating. Ind. Crops Prod. 2015, 66, 194-205. [CrossRef]

8. Soykeabkaew, N.; Thanomsilp, C.; Suwantong, O. A review: Starch-based composite foams. Composites Part A Appl. Sci. Manuf. 2015, 78, 246-263. [CrossRef]

9. Biliaderis, C.G.; Maurice, T.J.; Vose, J.R. Starch gelatinization phenomena studied by differential scanning calorimetry. J. Food Sci. 1980, 45, 1669-1674. [CrossRef]

10. Kozlowski, M.; Masirek, R.; Piorkowska, E.; Gazicki-Lipman, M. Biodegradable blends of poly(L-lactide) and starch. J. Appl. Polymer. 2007, 105, 269-277. [CrossRef]

(C) 2019 by the authors. Licensee MDPI, Basel, Switzerland. This article is an open access article distributed under the terms and conditions of the Creative Commons Attribution (CC BY) license (http://creativecommons.org/licenses/by/4.0/). 